\title{
L’infra-ordinaire : esquisse de la théorie narrative de Georges Perec
}

\author{
Julie ZAMORANO \\ Université Paris-Sorbonne \\ juliezamorano@gmail.com
}

Recibido: 31/10/2014

Aceptado: 09/01/2015

\begin{abstract}
Résumé
Georges Perec est l'auteur de romans autant que de nombreux écrits non fictionnels, de type essayistique, qui retiennent ici notre attention. À partir de la notion d'infra-ordinaire en tant qu'observation attentive de « ce qui se passe chaque jour et revient chaque jour, le banal, le quotidien, l'évident, le commun, l'ordinaire, l'infra-ordinaire, le bruit de fond, l'habituel », cette étude a pour objet de se pencher sur les écrits critiques de Perec pour en dégager la généalogie de ce qui sera son ébauche d'une théorie narrative. En parcourant les idées développées dans les textes non fictionnels de l'écrivain, l'attention portée aux détails du réel apparaît comme une constante qui finira par devenir une théorie rassemblant la pensée et la pratique littéraires de l’auteur.
\end{abstract}

Mots clés : Georges Perec, théorie narrative, textes critiques, infra-ordinaire, quotidien, habituel.

\section{Lo infraordinario: esbozo de la teoría narrativa de Georges Perec}

\begin{abstract}
Resumen
Georges Perec es autor de novelas tanto como de numerosos escritos no ficcionales, de tipo ensayístico, que son el objeto de nuestro estudio. Partiendo de la noción de infraordinario entendida como la atenta observación de « lo que sucede a diario y se repite a diario, lo banal, lo cotidiano, lo evidente, lo común, lo ordinario, lo infraordinario, el ruido de fondo, lo habitual », este artículo pretende analizar los escritos críticos de Perec para extraer la genealogía de lo que se convertirá en su esbozo de una teoría narrativa. Al recorrer la ideas desarrolladas en los textos no ficcionales del autor, la atención prestada a los detalles de la realidad aparece como una constante que acabará por convertirse en una teoría literaria que contiene el pensamiento y la práctica literarios del escritor.
\end{abstract}

Palabras clave: Georges Perec, teoría narrativa, textos críticos, infra-ordinario, cotidiano, habitual.

\section{The infra-ordinary: a draft of Georges Perec’s narrative theory}

\begin{abstract}
Georges Perec wrote novels as well as of non-fictional texts, which are precisely the object of this study. Starting from the notion of infra-ordinary conceived as the attentive observation of " what happens every day and repeats every day, what is banal, daily, evident, common, ordinary, infraordinary, background noise, habitual », this article analyses Perec's critical texts in order to bring out the genealogy of what will become his draft for a narrative theory. By following the ideas developed
\end{abstract}


in his non-fictional writings, the attention given to the details in reality reveals itself as a constant theme that will become a literary theory that combines Perec's practice and views on literature.

Key words: Georges Perec, narrative theory, critical texts, infra-ordinary, daily, ordinary.

Sommaire : 1. La Ligne générale. 2. L’infra-ordinaire. 3. Espace et écriture : les lieux de l'infraordinaire. 4. Une théorie de l'infra-ordinaire.

\section{Referencia normalizada}

Zamorano, J. (2015). "L'infra-ordinaire: esquisse de la théorie narrative de Georges Perec ». Thélème. Revista Complutense de Estudios Franceses, Vol. 30, Núm. 2: 269-282. http://dx.doi.org/10.5209/rev_THEL.2015.v30.n2.47021

Georges Perec est très souvent étudié pour ses œuvres narratives, mais il a également publié des textes de critique sur la littérature de son époque ainsi que sur ses propres œuvres. Ses essais sont tout aussi dignes d'attention car ils tiennent une place déterminante dans l'ensemble de son œuvre. De fait, Perec a porté l'habit de l'essayiste pendant sa jeunesse, habit qu'il délaissera au profit d'une écriture plus tournée vers le romanesque, mais qui, cependant, ne le quittera jamais véritablement. Si bien le romancier avouait n’avoir « jamais été à l’aise pour parler d'une manière abstraite, théorique, de [s]on travail » (Perec, 2003a : 11), il n'en reste pas moins qu’il a porté un regard théorique sur sa propre écriture qui débouchera sur un début de théorie narrative intrinsèquement liée à la notion d'infra-ordinaire. C'est à partir de ses premières incursions dans la critique de certains nouveaux romanciers (Robbe-Grillet en particulier, mais aussi Nathalie Sarraute [Perec, 1992b : 25-45]) qu’il abordera la littérature des autres, et la sienne par la suite, d'un point de vue théorique. Il sera donc question à présent d'étudier ses origines théoriciennes et leur rôle dans la mise en place d'une esthétique de «l'infra-ordinaire », ainsi que les relations entre sa pratique d'écrivain et cette notion qui s’avère être à la source de ce qui deviendra l'ébauche de sa théorie narrative.

\section{La Ligne générale}

Si l'on observe la bibliographie des ouvrages perecquiens élaborée par Bernard Magné, la plupart des textes publiés de 1955 à 1964 sont majoritairement des comptes-rendus de livres ou bien des articles de critique. Pendant cette même période, de 1959 à 1963, Perec fait partie du projet d’une revue qui ne vit jamais le jour, nommée La Ligne générale, mais qui permit à l'écrivain de s'exercer à la 
critique et à la réflexion théoriques ${ }^{1}$. De fait, ainsi que le remarque Claude Burgelin, cette expérience aura laissé chez l'auteur un regard critique qui le ne quittera plus :

La Ligne générale a été un moment déterminant de l’itinéraire de Perec. Il a trouvé là un milieu, une chauffe intellectuelle. Il a pu y affirmer ses choix littéraires, les étayer par un grand nombre de lectures, les faire passer au crible de formalisations théoriques, inaugurer des amitiés stimulantes (Burgelin, $1988: 238)^{2}$.

En effet, mis à part les articles recueillis dans le volume L.G. Une histoire des années soixante clairement présentés comme des textes de critique, Perec a constamment publié des ouvrages de type essayistique. Si à partir de 1965, année de parution des Choses, il se concentre plus sur l'écriture de romans, il consacre tout de même une partie de son activité scripturale à des essais. Ainsi, Espèces d'espaces paraît en 1974 dans la collection «L'espace critique » chez Gallimard, attestant par là de son caractère de texte critique (Magné, 1993 : 46) ${ }^{3}$. De même, les recueils posthumes L'infra-ordinaire, Penser/Classer et Je suis né regroupent des articles parus dans des journaux ou des revues de manière disséminée, dans lesquels sont développées diverses réflexions littéraires, pour la plupart en lien avec sa propre pratique d'écrivain. Chaque texte étant relié, d'une manière ou d'une autre, à des considérations sur la littérature, à un auteur, à un projet de livre ou encore à son rapport à l'écriture, la réflexion sur la littérature à laquelle se livre l'auteur dans ces différents textes le conduira dans ses dernières années à commencer l'élaboration de sa propre théorie de la narration. Comme il sera développé par la suite, cette esquisse d'une théorie se révèle être étroitement liée à certaines idées sur la littérature réaliste de l'époque de La Ligne générale qui, bien que modifiées au cours du temps, resteront un des noyaux de la conception de la littérature de Perec :

Mise à l'épreuve par l'écriture et par les recherches formelles dans lesquelles Perec s'engagera par la suite, sa conception d'une littérature réaliste formulée au début des années soixante subira des modifications considérables. Mais, sous une forme ou une autre, la volonté tenace de dire le réel restera l'une de ses préoccupations majeures (Van Montfrans, 1999 : 3).

Si l'échec de la revue le conduit à modifier sa conception de la littérature, il n'en demeure pas moins que l'approche théorique et le lien entre littérature et réel sont constants au long du parcours de l'écrivain. Pendant la période de La Ligne

\footnotetext{
${ }^{1}$ Si la revue ne fut jamais imprimée, il n'en reste pas moins que, le temps du projet, Perec aura écrit plusieurs articles publiés pour la plupart dans la revue Partisans, puis réunis de manière posthume dans le recueil L.G. Une aventure des années soixante (1992).

${ }^{2}$ Il faut ajouter que ce regard critique était déjà présent chez Perec qui, entre 1955 et 1959, bien que de manière irrégulière, a publié des notes de lecture dans la Nouvelle N.R.F et dans Les Lettres nouvelles. Se reporter pour plus de détails à l'ouvrage de Magné (1993).

${ }^{3}$ Autre preuve à l'appui, l'ouvrage de Michael Sheringham (2013: 61-73), Traversées du quotidien, Paris, PUF, où Espèces d'espaces est analysé à la lumière de la théorie sur l'essai formulée par Th. Adorno.
} 
générale, placée sous l’influence des travaux de Lukács ${ }^{4}$, la littérature pour Perec doit être non pas une manière de décrire une réalité qui resterait toujours extérieure à l'œuvre, mais une forme d'art faisant partie de la réalité à laquelle elle donne une forme et, par conséquent, une cohérence et un sens : « le réalisme est description de la réalité, mais décrire la réalité c'est plonger en elle et lui donner forme, c'est mettre à jour l'essence du monde : son mouvement, son histoire " (Perec, 1992b : 51). Son approche du réel sera quelque peu modifiée par la suite à partir des travaux de Barthes et de Lefebvre sur le quotidien, à partir desquels il pourra développer et s'approprier la notion d' "infra-ordinaire » qui deviendra un véritable système d'écriture.

\section{L'infra-ordinaire}

La notion d' « infra-ordinaire » est évoquée comme telle pour la première fois sous la plume de Perec en 1973 dans « Approches de quoi ? » (Perec, 1989a : 9-13), paru dans la revue Cause Commune, fondée par Georges Perec, Paul Virilio et Jean Duvignaud. C'est dans ce texte s'apparentant à un manifeste qu'est décrit le principe de cette notion qui consiste en l'observation attentive et la notation de « ce qui se passe chaque jour et revient chaque jour, le banal, le quotidien, l'évident, le commun, l'ordinaire, l'infra-ordinaire, le bruit de fond, l'habituel » (Perec, 1989a : 11). Pourtant, déjà en 1966 et 1967, il avait publié de petits textes « dit[s] d’humeur sur des sujets plus ou moins d'actualité. La série s'appelait, je crois, "l'esprit des choses" " "(Perec, 1985 : 319), parus dans le magasine Arts et Loisirs. Ces textes, dont les titres parlent par eux-mêmes ( «La Rolls-Royce », " Les idées du jour », « Éloge du hamac », « Le Hit-Parade », « Le vrai petit bistrot » [Magné, 1993 : 24]), l'avaient déjà mis sur la piste d'une pensée du quotidien, d'une attention aux objets du commun.

Cette réflexion sur l'habituel n'est toutefois pas du seul fait de Perec, ainsi que le démontre Derek Schelling (2006) dans son ouvrage Mémoires du quotidien : les lieux de Perec. D’une part, un des objectifs de la revue Cause Commune était de faire une " "anthropologie de l'homme contemporain" assortie d'une "investigation de la vie quotidienne à tous ses niveaux dans ses replis ou ses cavernes généralement dédaignés ou refoulés” » (cité par Schilling, 2006 : 54). C’est dans cette perspective qu'en février 1973, un numéro est consacré précisément à la

\footnotetext{
${ }^{4}$ Dans la préface de Claude Burgelin au volume, ce dernier affirme l'influence de la pensée de Lukács sur l'esprit recherché par la revue : " Cette croyance en un apprentissage à la fois conflictuel et méthodique de la réalité, cette affirmation du pouvoir de la raison critique et de la dialectique doivent à l'évidence autant à Sartre [...] qu'à Eisenstein, Brecht ou -surtout- Lukacs (sic) » (Burgelin, 1992 : 15).

${ }^{5}$ La rubrique dont Perec parle est effectivement intitulée « L'esprit des choses » (Brasseur, 2003 : 329). Cet « esprit des choses » est à mettre également en perspective avec le roman Les Choses publié en 1965. Se référer à ce propos au chapitre 35 de la biographie de Perec (Bellos, 1994 : 373-376).
} 
question de l' « infra-ordinaire » ou l' « infra-quotidien » (terme dont la genèse est attribuée à Paul Virilio [Duvignaud, 1993 : 51]), numéro dans lequel paraît le texte programmatique de l'œuvre perecquienne « Approches de quoi ? ».

D'autre part, la question du quotidien est présente dans divers milieux intellectuels de l'après-guerre jusqu'aux environs des années 1980, en raison du changement d'un régime historique à un régime social dans les sciences humaines :

Entre 1945 et 1980, années où les sciences humaines effectuent une transition clef du régime historique (le fait singulier inséré dans une logique causale) au régime social (le fait récurrent susceptible d'explication structurale), historiens, sociologues, philosophes et anthropologues ont fait appel aux notions de " quotidien ", « quotidienneté » et " vie quotidienne " pour identifier un niveau d'analyse lié à la réalité sociale que le développement interne de leurs disciplines empêchait de reconnaître. [...] Les «Grands récits » dont parle Jean-François Lyotard ne fonctionnant plus guère, commence le règne de la vie quotidienne, avec tout ce qu'il implique de pauvreté et de richesse (Shilling, $2006:$ 19-20).

La centralité de cette notion dans différentes disciplines est à mettre également en perspective avec l'avènement de la société de consommation et la charge symbolique (de richesse ou de pauvreté) attribuée aux objets. Les "Trente Glorieuses » seront ainsi l'occasion de nombreux travaux sur la question de la quotidienneté, dont les œuvres Mythologies (1957) de Roland Barthes et Critique de la vie quotidienne I, II et III (1947-1961-1981) d'Henri Lefebvre figurent parmi les plus représentatives.

De fait, non seulement ces auteurs ont considérablement imprimé l'esprit des années 1960, mais ils ont aussi fortement influencé la pensée de Perec, qui a assisté aux séminaires du premier et a participé au " groupe d'étude de la vie quotidienne » du second (Bellos, $1994: 255)^{6}$. L'influence de Barthes sur Perec se trouve plutôt du côté de la rhétorique publicitaire qui sera, par ailleurs, à l'origine du roman Les Choses : «à l'origine de ce livre, il y avait un projet double : d'abord un exercice sur les Mythologies de Barthes, c'est-à-dire sur le reflet en nous du langage publicitaire ; ensuite la description aux traits à peine appuyés d'un certain milieu, qui d'ailleurs est le mien » (Bertelli, 2003a : 48). Quant à ses liens intellectuels avec la pensée de Lefebvre, Perec s’en détache par une dépolitisation de son approche de la quotidienneté. Si la réflexion de Lefebvre est fondamentalement centrée sur la possibilité d'une libération de l'aliénation -due à la programmation et au façonnement des besoins- par un travail d'élucidation et de transformation du quotidien ${ }^{7}$, l'approche de Perec vise la transformation du regard sur ce quotidien ${ }^{8}$,

\footnotetext{
${ }^{6}$ Pour une analyse plus approfondie des liens conceptuels entre Perec, Barthes et Lefebvre, se référer respectivement à l'article de Mireille Ribière (2005: 338-353), ainsi qu'aux ouvrages de Sheringham (2013) et de Schilling (2006).

7 «Bien aménagé, [le quotidien] tend à constituer un système avec un bouclage propre (production-consommation-production). [...] Pour briser le cercle vicieux infernal, pour empêcher le bouclage, il ne faut rien de moins que la conquête de la quotidienneté par une série d'actions -
} 
notamment à travers le travail de la description de ce qui passe inaperçu. Il s’agit ainsi de faire émerger une rhétorique de l'habituel pour le rendre inhabituel et inciter de la sorte à voir autrement, à interroger l'ordinaire et le banal pour tenter de saisir ce qu'il peut révéler de notre vérité ${ }^{9}$ :

Il m’importe peu que ces questions soient, ici, fragmentaires, à peine indicatives d'une méthode, tout au plus d'un projet. Il m'importe beaucoup qu'elles semblent triviales et futiles : c'est précisément ce qui les rend tout aussi, sinon plus, essentielles que tant d'autres au travers desquelles nous avons vainement tenté de capter notre vérité (Perec, 1989a : 13).

L' « infra-ordinaire » peut en ce sens être considéré comme la mise en pratique de cette idée qui lui venait de l'époque de La Ligne générale d’une littérature susceptible de capter le réel dans sa vérité. L’idée d’une littérature réaliste en germe dans la conception littéraire de Perec depuis le milieu des années 1950 prendra la forme de l'infra-ordinaire dans le texte de 1973, et sera l'un des piliers de sa littérature : «Le motif court tout au long de son œuvre, des Choses (1965) à La Vie mode d'emploi (1978) et jusqu'à ses derniers projets [...]» (Sheringham, 2013 : 257). En plus d'être un motif constant dans les œuvres narratives de Perec, il est également exploité dans les essais, en particulier dans Espèces d'espaces où les lieux, considérés à partir de l'infra-ordinaire, se révèlent être des lieux de mémoire ${ }^{10}$.

investissements, assauts, transformations- à mener aussi selon une stratégie. La suite seulement dira si nous (ceux qui voudront) retrouverons ainsi l'unité entre le langage et la vie réelle, entre l'action qui change la vie et la connaissance » (Lefebvre, 1968 : 141-142).

${ }^{8}$ La place qu’occupe le regard dans l'écriture de Perec a été remarquablement analysée dans le chapitre «Histoire de l’œil » (Heck, 2012 : 52-74). Le déplacement du regard, le changement de perspective persiste jusqu'au début des années 1990, par exemple chez Jean-Didier Urbain (1993 [1991]) : 225) qui nomme "voyageur de l'interstice " « ce touriste qui, au cœur de l'espace connu ou quotidien, réinvente le regard distancié nécessaire à l'expérience de l'étrangeté et au plaisir de la découverte ». En italiques dans le texte.

${ }^{9}$ Cette démarche de transformation du regard est également présente du côté de la création artistique, notamment chez les surréalistes qui voyaient dans les objets du commun de civilisations passées (les « trouvailles ») la possibilité de reconstituer leur histoire.

${ }^{10}$ Depuis l'Antiquité, mémoire et espace sont étroitement liés comme le démontre le système mnémonique de la rhétorique tel que décrit dans l’Ad Herennium libri $I V$, ainsi que par Cicéron et Quintilien (Yates, 1975 [1966] :14). En effet, le travail de la mémoire consiste en un système d'association de lieux et d'images afin d'organiser les souvenirs pour mieux les retrouver : " [L'art de la mémoire] était une technique qui permettait à l'orateur d'améliorer sa mémoire, qui le rendait capable de prononcer de longs discours de mémoire, avec une précision impeccable. [...] dans l'Antiquité, qui ignorait l'imprimerie, qui n'avait pas de papier pour prendre des notes ou pour taper à la machine des conférences, une mémoire exercée avait une importance vitale. Et la mémoire des anciens était exercée à l'aide d'un art qui reflétait l'art et l'architecture du monde antique [...] » (Yates, 1975 [1966] : 14-16). 


\section{Espace et écriture : les lieux de l’infra-ordinaire}

La volonté d'apprendre à regarder la réalité autrement afin de révéler «notre vérité » sera mise à l'œuvre dans Espèces d'espaces, où Perec applique sa méthode rigoureuse d'observation à la manière dont nous habitons l'espace. L'objectif d'une telle démarche est « d'interroger, ou, plus simplement encore, de le lire ; car ce que nous appelons quotidienneté n'est pas évidence, mais opacité » (Perec, 2010 [1974] : Prière d'insérer). Partant du fait que le livre est présenté comme le " journal d'un usager de l'espace » (Perec, 2010 [1974] : Prière d'insérer) et que cet usager est un écrivain, les liens qui seront faits avec la littérature apparaissent dès lors comme inéluctables. L'ouvrage est à considérer comme un essai dans la mesure où il contient des réflexions subjectives sur son rapport littéraire à l'espace ainsi que sur les liens entre espace et écriture : "Le sujet perecquien se définit à partir de son espace. Aussi bien des lieux géographiques précis que l'espace littéral du livre, celui de la page » (Sirvent, 2007 : 31). De fait, suivant un mouvement qui décrit l'espace en partant du plus petit au plus grand (allant de la page, le lit, la chambre, l'appartement, l'immeuble, la rue, le quartier, la ville, la campagne, le pays, l'Europe, le monde, jusqu'à l'espace de l'univers ${ }^{11}$ ), le livre développe une réflexion sur l'écriture associée à chacun des lieux évoqués.

Le premier type d'espace dont il est question est celui de la page en tant que lieu premier de l'écriture où viennent habiter les mots : "L'espace commence ainsi, avec seulement des mots, des signes tracés sur la page blanche » (Perec, 2010 [1974] : 26). La pensée sur l'espace débute dès lors par une réflexion sur le lieu même où elle advient, c'est-à-dire sur la page où se déploie l'écriture qui la fige et lui donne un sens. La partie suivante, " Le lit », est également associée à l'écriture à partir de la mise en relation du fait que le lit, comme la page, est généralement utilisé «dans le sens de sa plus grande dimension» (Perec, 2010 [1974] : 33). L'autre lien entre la littérature et le lit provient de la double lecture du mot « lit » : c'est une forme du verbe "lire » aussi bien qu'un synonyme de couchette en tant que lieu propice à la lecture : "C'est couché à plat ventre sur mon lit que j'ai lu Vingt ans après, L'île mystérieuse et Jerry dans l'île » (Perec, 2010 [1974] : 34). Bien que Perec n'évoque pas d'autre usage littéraire du lit, celui-ci est aussi un lieu d'écriture : l'on pense notamment à Proust qui a écrit la plus grande partie de A la recherche du temps perdu dans son lit.

C’est précisément Proust qui viendra alimenter le rapport entre l'écriture et la chambre dans la mesure où cette dernière fonctionne pour Perec "comme une madeleine proustienne » (Perec, 2010 [1974] : 47). La dimension littéraire de la chambre apparaît ici de deux manières : d’abord, la plus évidente, où la chambre est

\footnotetext{
${ }^{11}$ L'organisation du livre à partir d'une disposition croissante des lieux (de la page à l'univers) rappelle les pratiques rhétoriciennes de l'organisation de la mémoire à partir d'une visualisation spatiale. Voir à ce sujet Schilling (2006 : 143-147).
} 
associée à l'œuvre de Proust, c'est-à-dire qu'elle est perçue à partir d'une œuvre littéraire ; ensuite, en tant qu'elle constitue le thème central d'un des projets de Perec : Lieux où j'ai dormi, dont l'objectif était de se remémorer et de décrire toutes les chambres dans lesquelles il avait dormi. Même si ce projet n'a pas abouti, il n'en demeure pas moins que le lieu qu'est la chambre lui a servi de matériau d'écriture. À ce sujet, plusieurs lieux dont il est question dans Espèces d'espaces sont directement reliés à des projets d'écriture, donnant par là une dimension littéraire à la perception de l'espace. Tel est le cas du chapitre sur «l'immeuble » qui sera la matrice de La Vie mode d'emploi, où l'idée d'ensemble du roman y est décrite, tant au niveau de l'histoire que de la structure :

J'imagine un immeuble parisien dont la façade a été enlevée [...] de telle sorte que, du rez-dechaussée aux mansardes, toutes les pièces qui se trouvent en façade soient instantanément et simultanément visibles [...] le tout selon des processus formels dans le détail desquels il ne me semble pas nécessaire d'entrer ici, mais dont les seuls énoncés me semblent avoir quelque chose d'alléchant : polygraphie du cavalier (adaptée, qui plus est, à un échiquier de $10 \mathrm{x} 10$ ), pseudoquenine d'ordre 10, bi-carré latin orthogonal d'ordre 10 [...] (Perec, 2010[1974] : 81) ${ }^{12}$.

Le chapitre suivant, "La rue », fait apparaître ce lieu comme un des endroits privilégiés de l'infra-ordinaire. Dans ce chapitre figure une brève description de la méthode d'observation et d'écriture de « ce qui n'a pas d'intérêt, ce qui est le plus évident, le plus commun, le plus terne » (Perec, 2010 [1974] : 100). De plus, le chapitre présente la description d'un important projet né en 1969, Les Lieux, qui n'aboutira pas en tant que tel, mais dont la ligne directrice subsistera dans divers autres textes, dont le plus connu est Tentative d'épuisement d'un lieu parisien (2008 [1975]), mais aussi « Tentative d'inventaire des aliments liquides et solides que j'ai ingurgités au cours de l'année mil neuf cent soixante-quatorze » (Perec, 1989b : 97106). En 1977, paraissent trois autres textes dans la même lignée : "Tentative de description de quelques lieux parisiens: Guettées » (Perec, 1977a: 61-71), "Tentative de description de quelques lieux parisiens: vues d'Italie » (Perec, 1977b : 239-246), « La rue Vilin » (Perec, 1989c : 15-31) ${ }^{13}$.

Le projet Les Lieux, programmé sur douze ans, consistait en la description de douze lieux spécifiques, à raison de deux endroits deux fois par mois : la première fois la description se ferait sur place, la deuxième dans un autre endroit à partir d'un travail de remémoration (Perec, 2010 [1974]: 100). De plus, le choix des emplacements n'a rien d'anodin car il s'agit de lieux ayant une forte charge

\footnotetext{
${ }^{12}$ Pour une approche détaillée de la structure de La Vie mode d'emploi, se référer à Hartje, H., Magné, B. \& J. Neefs (ed.), (1993) Georges Perec. Cahier des charges de La Vie mode d'emploi. Paris-Cadeilhan, Éditions du CNRS-Zulma.

${ }^{13}$ La volonté d'épuisement descriptif est également transférée à d'autres types d'espaces, par exemple le bureau qui a donné deux textes de mise en pratique du regard acéré et de description minutieuse : « Notes concernant les objets qui sont sur ma table de travail » (Perec, 2003b) et « Still life/Style leaf » (Perec, 1989d).
} 
personnelle pour l'écrivain : « En 1969, j'ai choisi, dans Paris, 12 lieux (des rues, des places, des carrefours, un passage), ou bien dans lesquels j'avais vécu, ou bien auxquels me rattachaient des souvenirs particuliers" (Perec, 2010 [1974] : 108). Dans cette perspective, espace, mémoire et écriture sont entièrement imbriqués les uns dans les autres ce qui, au demeurant, était la volonté de l'écrivain qui cherchait à faire émerger « tout à la fois le vieillissement des lieux, le vieillissement de [s]on écriture, et le vieillissement de [s]es souvenirs " (Perec, 1990 : 59). La transformation de l'espace de l'infra-ordinaire en écriture se fait lieu de mémoire, ici personnelle, mais qui deviendra par la suite le recensement d'une mémoire collective ainsi qu'il le fera dans Je me souviens.

Entre 1976 et 1978, cette pratique de recensement de l'habituel sera expressément appliquée à la mémoire collective dans Je me souviens, livre dans lequel Perec s'efforce de faire un inventaire des souvenirs communs à sa génération qui ne soient pas nécessairement des événements historiques, mais plutôt des souvenirs de choses ordinaires qui permettraient de révéler l'esprit d'une époque. Se plaçant dans la perspective de M. Sheringham, Espèces d'espaces et Je me souviens semblent deux textes issus du projet Les lieux où « les fragments de Je me souviens se nourrissent du réseau des "souvenirs", tandis qu'Espèces d'espaces serait une extension du propos des textes "réels" » (Sheringham, $2013: 272$ ) -par « réel » s'entend les textes écrits sur place et par «souvenirs » ceux écrits de mémoire.

Ainsi, il apparaît que la pratique descriptive du projet Les Lieux ainsi que la mise en relation entre écriture, mémoire et interrogation du quotidien mise à l'œuvre dans Espèces d'espaces proviennent directement de la notion d'infra-ordinaire qui peut être envisagée comme la source de l'esquisse de la théorie de la narration élaborée par Perec durant ses dernières années de vie.

\section{Une théorie de l'infra-ordinaire}

Pour Perec, écrire c'est avant tout être un «homme de lettres », c'est-à-dire un homme qui travaille avec les lettres de l'alphabet. C'est donc à partir de la matérialité de l'écriture, de ce qui peut apparaître " ordinaire » comme peuvent l'être les lettres de l'alphabet, qu’advient la littérature : «L'aleph, ce lieu borgésien où le monde entier est simultanément visible, est-il autre chose qu'un alphabet ? " (Perec, 2010 [1974] : 26). L’attention portée aux détails est donc transposée à la littérature qui serait l'ensemble formé par les mots et les lettres dans leur matérialité, dans leur ordinarité. Cette façon de concevoir l'écriture donnera naissance à une ébauche de ce qui aurait pu devenir sa théorie narrative, mais qu'il n’a pas eu le temps d'achever, la mort l'emportant à 46 ans. Cette théorie, que l'on pourrait nommer « théorie de l’infra-ordinaire », apparaît de manière rétrospective comme la mise en théorie de tout son système d'écriture :

Je commence juste... depuis quelques semaines, à formuler quelque chose qui pourrait devenir un jour, un jour lointain, une théorie de la narration. Je ne peux que vous en donner un exemple. Vous savez, en linguistique, les gens parlent de morphème et de lexème, et j'en suis venu à penser 
qu'il y a quelque chose que l'on pourrait appeler un narratème. C’est un élément minuscule qui... comme la perle à l'intérieur de l'huître, pourrait progressivement devenir le roman, le récit. Au début, il n’y a presque rien. Je pense que c’est précisément ce que fait Balzac. Vous voyez, il commence à écrire un livre, deux livres, trois livres, et ensuite un des personnages réapparaît dans un autre livre puis dans un autre livre et, à la fin, la masse des livres ressemble à la description de tout le XIX ${ }^{\mathrm{e}}$ siècle (Bertelli, 2003b : 257-258).

La démarche décrite ici par l'écrivain ressemble singulièrement à l'idée que l'infra-ordinaire serait susceptible de révéler quelque chose de notre vérité et que, de la même manière, le narratème, l'unité la plus petite de la narration, le détail à partir duquel toute l'écriture peut se déployer, deviendrait par un effet d'expansion la description de tout un siècle. Tant dans sa manière d'appréhender le monde que dans sa manière de concevoir l'écriture, le détail se fait la clef pour aboutir à une image d'ensemble. À ce titre, en mettant en perspective cette esquisse théorique avec l'image du puzzle si chère à Perec, les deux notions semblent s'éclairer l'une l'autre du fait que le puzzle fonctionne comme une métaphore de la littérature :

Il y a en ce qui me concerne une image de la littérature qui se dessine et qui serait l'image d'un puzzle. [...] Butor a expliqué que tout écrivain est entouré par une masse d'autres [...] écrivains [...] et ce puzzle qui est la littérature, dans l'esprit de cet écrivain, a toujours une place vacante, c'est évidemment celle que l'œuvre qu'il est en train d'écrire va venir remplir (cité par Van Montfrans, 1999 : 69).

C’est-à-dire que, de la même manière que la pièce du puzzle contribue à élaborer l'image d'ensemble, la réduction de la littérature à sa plus petite forme, ici le narratème, ne prend son sens que dans la perspective de la description d'un siècle entier. En ce sens, le narratème serait à la littérature ce que l'infra-ordinaire est à la réalité dans la conception perecquienne. L'importance de la partie pour former un tout, l'attention portée au détail comme révélateur de l'ensemble, telles sont les idées qui semblent ici guider le schéma de sa théorie. De la même manière que son approche du quotidien est supposée révéler la vérité d’une société, la littérature est une somme de narratèmes dont l'observation et l'assemblage seraient une description de l'ensemble d'un siècle. En effet, la démarche de description de l'infra-ordinaire dérive d'une démarche premièrement personnelle, individuelle vers une démarche de recensement d'une mémoire collective : " Mettre en question les évidences de la vie de tous les jours, décrire le vécu au cœur de son émergence, rendre compte des pratiques de chacun, en un mot épuiser par écrit le réel afin de constituer une archive à l'usage des générations aussi bien présentes que futures [...] » (Schilling, 2006 : 16). La brève esquisse de la théorie de la narration de Perec permet de considérer le narratème littéraire comme l'équivalent de l'infra-ordinaire du réel qui permettrait de passer d'une mémoire personnelle à une mémoire collective, d'une histoire individuelle qui permettrait de faire émerger une histoire collective. En ce sens, l'ébauche de sa théorie narrative apparaît comme l'aboutissement théorique, comme la mise en système de sa pratique d'écrivain qui consiste à partir du minuscule pour atteindre une vue d'ensemble et ainsi parvenir à une œuvre qui se voudrait totalisante : « quel que soit l'assemblage, ce qui demeure 
important à l'horizon têtu de l'œuvre, c'est l'ambition ou la volonté de totalité » (Joly, 2011 : 26).

Établir un lien de continuité dans toute l'œuvre perecquienne peut être une entreprise risquée si l'on ne tient pas en compte la rupture qu'implique son entrée à l'Oulipo en 1967. Toutefois, l'existence d'un fil rouge qui traverserait tout son travail d'écrivain et aboutirait à sa théorie (plutôt qu'à une théorie) de la narration a été formulée par Perec lui-même : "je sens confusément que les livres que j'ai écrits s'inscrivent, prennent leur sens dans une image globale que je me fais de la littérature » (Perec, 2003a : 12). Partant de l'idée qu'il y a une certaine conception de la littérature qui persiste tout au long de son écriture, chacune de ses œuvres peut également être envisagée comme chacune des pièces du puzzle qu'est la description de la société parisienne des années 1960-1980 : Les Choses (1965) a pour sujet la vie d'un couple entièrement habité par l'esprit consommateur des années soixante ; Quel petit vélo...? (1966) parle de la jeunesse mobilisée pendant la guerre d'Algérie ; Un homme qui dort (1967) envers des Choses, pose la question du refus de la société de consommation; $W$ ou le souvenir d'enfance (1975) parle de manière oblique des enfants de la Seconde Guerre mondiale ; Je me souviens (1978) vise à renfermer les souvenirs de toute une génération; La Vie mode d'emploi (1978) contient dans un seul immeuble des centaines d'histoires et de personnages de divers milieux sociaux vivant dans un Paris de la fin des années 1970. Ce livre, sommet de cette entreprise descriptive, la contient toute dans le titre aussi bien que dans l'immeuble. Expliquant la signification du titre, il exprime le caractère éminemment descriptif de cet ouvrage :

il s'agit d'une opposition entre la vie -le désordre, la prolifération - et l'emploi : le fait de trouver une règle, un ordre qui ne fonctionne pas, illusoire donc. La vie effervescente, chaotique, opposée à l'ordre que nous croyons mettre. Mais il ne s'agit pas d'une leçon de vie, non, plutôt d'une tentative de description qui m'a demandé dix ans de travail (Bertelli, 2003b : 84-85) ${ }^{14}$.

Il apparaît ainsi que la réflexion sur la littérature initiée dans les articles de jeunesse, bien que considérablement modifiée au fil du temps, persistera sous la forme de l'infra-ordinaire et sera mise à l'œuvre dans ses romans. S'il y a chez lui une certaine gêne et même un refus de faire des discours théoriques sur son écriture $^{15}$, cela ne signifie pas pour autant qu'il n’y ait pas de réflexion théorique sur son travail. La manière dont il produit un discours "abstrait", c'est, paradoxalement, de manière concrète, c'est-à-dire dans ses œuvres narratives ; de sorte que la fiction devient le lieu même de la réflexion à travers la mise en œuvre de sa conception de la littérature :

${ }^{14}$ Je souligne.

${ }^{15}$ En 1979, Perec répondait fermement dans un entretien à la question de savoir s'il avait une théorie de la littérature et quelle était sa fonction : «Je n’ai pas de théorie et je n'ai pas de réponse » (Bertelli, 2003b : 86). Ce n’est qu'en 1981 qu’il ébauche son début de théorie. 
je crois plutôt trouver -et prouver- mon mouvement en marchant : de la succession de mes livres naît pour moi le sentiment, parfois réconfortant, parfois inconfortable (parce que toujours suspendu à un « livre à venir ", à un inachevé désignant l'indicible vers quoi tend désespérément le désir d'écrire), qu'ils parcourent un chemin, balisent un espace, jalonnent un itinéraire tâtonnant, décrivent point par point les étapes d'une recherche dont je ne saurais dire le " pourquoi » mais seulement le « comment » [...] (Perec, 2003a : 11-12).

Les romans parcourent donc le chemin de l'adéquation entre écriture et réel. Ce chemin, parti de la réflexion critique sur les œuvres des autres, aura conduit Perec à mettre en œuvre sa conception de la littérature puis, en fin de parcours, à en faire sa propre théorie. Selon une proposition d'Yvonne Goga, il y aurait, en amont de l'écriture, une théorie de la littérature qui donnerait lieu à une mise en pratique : «La création de la conscience artistique suppose [...] trois exigences principales, que l'on retrouve chez Perec: l'élaboration d'une théorie, la recherche d'une technique et leur mise en pratique » (Goga, 2007: 129). Cependant, il faudrait nuancer cet itinéraire, car la théorie précédant à l'écriture n'en est pas une tout à fait élaborée, car c'est l'écriture même qui lui permettra de théoriser ensuite son système ; ce sont ses œuvres en tant que telles qui lui permettront de faire émerger sa conception théorique de la littérature, une conception qui ne sera malheureusement jamais menée à bout. Le travail de réflexion ne se fait donc pas en dehors de l'écriture, mais bien en son intérieur, faisant de la fiction le terrain même de la théorie.

La centralité de la notion d'infra-ordinaire dans la pensée et l'écriture de Perec semble aller au-delà du seul domaine de la littérature et peut être considérée comme ayant une valeur existentielle, dans la mesure où l'observation et l'enregistrement du quotidien étaient une manière non seulement d'écrire, mais aussi de vivre et d'appréhender son propre quotidien. L'infra-ordinaire aura été sa manière d'écrire sa réalité.

\section{REFERENCES BIBLIOGRAPHIQUES}

Bellos, D., (1994) Georges Perec. Une vie dans les mots. Paris, Seuil.

Beaumatin, E. \& M. Ribière (dir.) (2005), De Perec etc., Derechef. Nantes, Joseph K., coll. « Essais ».

Bertelli, D. \& M. Ribière (ed.), (2003a) Georges Perec. Entretiens et conférences, vol. I, 1965-1978. Nantes, Joseph K.

Bertelli, D. \& M. Ribière (ed.), (2003b) Georges Perec. Entretiens et conférences, vol. II, 1979-1981. Nantes, Joseph K.

Brasseur, R., (2003) Je me souviens encore mieux de Je me souviens. Notes pour Je me souviens de Georges Perec à l'usage des générations oublieuses et de celles qui n'ont jamais su. Bègles, Le Castor Astral.

Burgelin, C., (1988) Georges Perec. Paris, Seuil. 
Burgelin, C., (1992) "Préface" in Perec, G., L.G. Une aventure des années soixante. Paris, Seuil, coll. « La librairie du $\mathrm{XX}^{\mathrm{e}}$ siècle », pp. 7-23.

Duvignaud, J., (1993) Perec, ou la cicatrice. Arles, Actes Sud.

Goga, Y., (2007) «Formes de l'autoréflexivité mallarméenne dans Un homme qui dort de Georges Perec » in Reggiani, C. \& B. Magné (dir.), Écrire l'énigme. Paris, PUPS, pp. 127-138.

Joly, J-L., (2011) « Des choses qui dorment » in Roman 20-50, n 51, juin 2011, pp. 11-26.

Lefebvre, H., (1968) La vie quotidienne dans le monde moderne. Paris, Gallimard, coll. « Idées ».

Magné, B., (1993) Tentative d'inventaire pas trop approximatif des écrits de Georges Perec. Bibliographie. Toulouse, Presses Universitaires du MirailToulouse, coll. « Les cahiers de Littératures ».

Perec, G., (2010 [1974]) Espèces d'espaces. Paris, Galilée.

Perec, G., (2008 [1975]) Tentative d'épuisement d'un lieu parisien. Paris, Christian Bourgois.

Perec, G., (1977a) «Tentative de description de quelques lieux parisiens: Guettées » in Les lettres nouvelles. № 1, pp. 61-71.

Perec, G., (1977b) «Tentative de description de quelques lieux parisiens : vues d'Italie » in Nouvelle Revue de Psychanalyse. $\mathrm{N}^{\circ} 16$, pp. 239-246.

Perec, G., (1978) Je me souviens. Paris, Hachette/POL.

Perec, G., (1985) «Bibliographie approximative » in Magné, B. (dir.), Cahiers Georges Perec 1. Colloque de Cerisy, Paris, P.O.L., pp. 307-323.

Perec, G., (1989a) « Approches de quoi ? » in L'Infra-ordinaire. Paris, Seuil, coll. « Librairie du XXI siècle », pp. 9-13.

Perec, G., (1989b) « Tentative d'inventaire des aliments liquides et solides que j'ai ingurgités au cours de l'année mil neuf cent soixante-quatorze » in L'Infraordinaire. Paris, Seuil, coll. « Librairie du XXI ${ }^{\mathrm{e}}$ siècle », pp. 97-106.

Perec, G., (1989c), "La rue Vilin », in L'Infra-ordinaire. Paris, Seuil, coll. « Librairie du XXI siècle », pp. 15-31.

Perec, G., (1989d) «Still life/Style leaf» in L'Infra-ordinaire. Paris, Seuil, coll. " Librairie du XXI ${ }^{\mathrm{e}}$ siècle », pp. 107-119.

Perec, G., (1990) « Lettre à Maurice Nadeau » in Je suis né. Paris, Seuil, coll. « La librairie du $\mathrm{XX}^{\mathrm{e}}$ siècle », pp. 51-66.

Perec, G., (1992a) L.G. Une aventure des années soixante. Paris, Seuil, coll. « La librairie du $\mathrm{XX}^{\mathrm{e}}$ siècle ».

Perec, G., (1992b) « Le Nouveau Roman et le refus du réel » in L.G. Une aventure des années soixante. Paris, Seuil, coll. « La librairie du XX $\mathrm{XX}^{\mathrm{e}}$ siècle », pp. 25-45.

Perec, G., (2003a) « Notes sur ce que je cherche » in Penser/Classer. Paris, Seuil, coll. « La librairie du XXI ${ }^{\mathrm{e}}$ siècle », pp. 9-12.

Perec, G., (2003b) « Notes concernant les objets qui sont sur ma table de travail » in Penser/Classer. Paris, Seuil, coll. « La librairie du XXI ${ }^{\mathrm{e}}$ siècle », pp. 17-23.

Reggiani, C., (2005) «Le roman de la théorie » in Beaumatin, E. \& M. Ribière (dir.), De Perec etc., Derechef. Nantes, Joseph K., coll. « Essais », pp. 328-337. 
Ribière, M., (2005) "Georges Perec, Roland Barthes : l'élève et le maître " in Beaumatin, E. \& M. Ribière (dir.), De Perec etc., Derechef.Nantes, Joseph K., coll. « Essais », pp. 338-353.

Schilling, D., (2006) Mémoires du quotidien : les lieux de Perec. Villeneuve d’Ascq, Presses Universitaires du Septentrion.

Sheringham, M., (2013) Traversées du quotidien. Paris, PUF.

Sirvent, M., (2007) Georges Perec ou le dialogue des genres. Amsterdam-New York, Rodopi.

Urbain, J.-D., (1993 [1991]) L'idiot du voyage. Histoires de touristes. Paris, Payot, coll. « Petite bibliothèque Payot/Documents ».

Van Montfrans, M., (1999) Georges Perec: la contrainte du réel. AmsterdamAtlanta, Rodopi, coll. « Faux titre ».

Yates, F., D. Arrasse (trad.), (1975 [1966]) L’art de la mémoire, Paris, Gallimard, coll. « Bibliothèque des histoires ». 